\title{
Spatial Fading Emulator for Base Station using Cavity-Excited Circular Array based on ESPAR Antenna
}

\author{
Chulgyun PARK, Jun-ichi TAKADA and Kei SAKAGUCHI \\ Graduate School of Science and Engineering \\ Tokyo Institute of Technology \\ 2-12-1, O-okayama, Meguro, Tokyo, 152-8552 JAPAN \\ Email: cgpark@ap.ide.titech.ac.jp
}

\author{
Takashi OHIRA \\ ATR Adaptive Communications Research Labs. \\ 2-2-2, Hikaridai, Keihanna Science City, \\ Kyoto, 619-0028 JAPAN \\ Email: ohira@atr.co.jp
}

\begin{abstract}
In this paper we propose a spatial fading simulator to evaluate performance of array antenna and show its computer simulation results based on parameters verified by experimental data. We introduce a cavity-excited circular array (CECA) as a spatial fading emulator that can simulate realistic mobile communicatoin environments. To evalute antenna array, two spatial stochastic characters is needed. The first one is the fading phenomenon and the second is the angular spread (AS) of incident wave. The computer simulation results on the two respects, fading and AS, show that CECA works well as a spatial fading simulator for evaluation of performance of antenna array. We first present the basic structure, features and design methodology of CECA, and then show computer simulation results on stochastic spatial charateristics with experimental parameters. The results convince us that it is a useful to evaluate performance of antenna array.
\end{abstract}

\section{INTRODUCTION}

Recently, in wireless communication field, the antenna array systems occupy an important position. To evaluate the antenna array systems, we make efforts at obtaining the reasonable results. However, the testing of mobile radio transmission techniques in the field is time-consuming and often inconclusive, due to uncertainty in the statistical signal variations actually encountered[1]. If we can reproduce the mobile communication environment at laboratory or anechoic chamber so that it provide the average mobile communication environment verified theoretically and observed experimentally, it may be another attractive alternative. Therefore, we need a standard mobile communication environment, which provides suitable condition for mobile radio transmission experiment. The received signal can be classified into two features, fading characteristics and spatial characteristics. The fading phenomena occurs when received signals become weak, below some level, instantaneously due to phase overlapping of waves coming through multi-path and this level variation follows Rayleigh distribution. In spatial characteristics, there are two properties, namely direction of arrival (DoA) and angular spread (AS). If we can emulate fading and AS that observed in a real mobile communication environment, it seems quite all right to consider that emulated environment to be close to the real

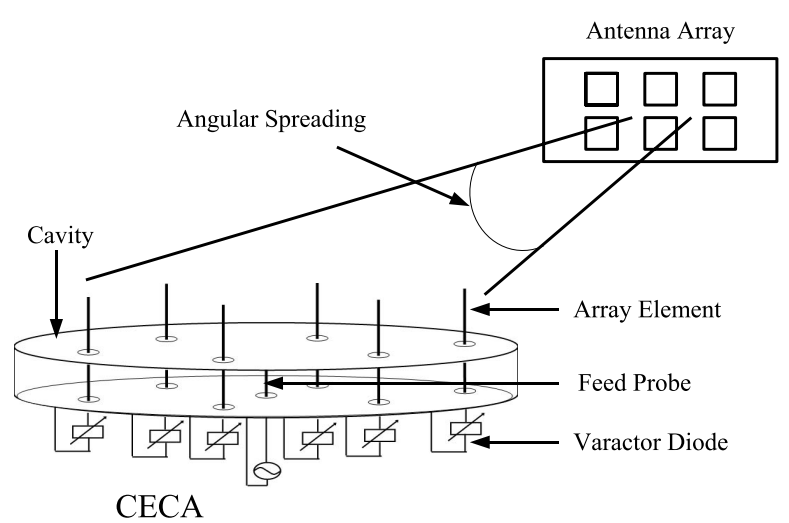

Fig. 1. Structure of CECA

mobile communication environment.

There are several approaches to generate fading signals. First is a stored channel approach in which actual fading fluctuations are stored in the memory[2]. Second is the so called "Jakes type" fading simulator in which a steady signal is split into several paths, each of which suffers from different Doppler shifts, and they are combined again to generate the fading[1]. Third is the Gaussian amplitude modulation of the in-phase and quadrature components of a steady carrier which can be used to provide uniform phase modulation and Rayleigh envelop fading[3].

The fading simulators can only work in the delay and Doppler domains, and the effect of antennas cannot be considered. To overcome this problem, a fading emulator called a field simulator has been proposed. For mobile terminals, a field simulator composed of a phased array antenna and the shielded box is used[4]. For base stations, another field simulator using the moving metal bars is used to realize the finite angular spread[5]. Yet another field emulator using electronically steerable passive array radiator (ESPAR) an- 


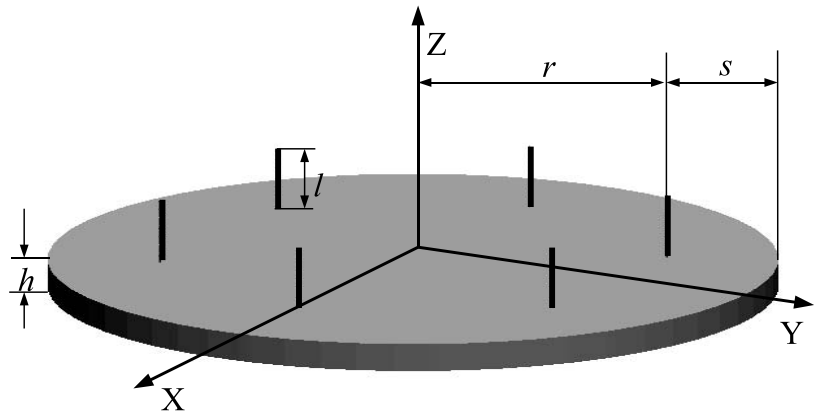

Fig. 2. Cartesian Coordinate in fabrication

tenna has recently been proposed by the authors[6][7]. ESPAR field simulator works well as a spatial fading emulator with low cost and simple structure but only a small AS could be generated under specific condition. Therefore a cavity-exited circular array (CECA) based on ESPAR antenna as a spatial fading emulator had been proposed to overcome small AS and severe conditions. The proposed structure uses a radial cavity as a feeding circuit so that the fundamental size limitation of an ESPAR antenna can be overcome, and we can obtain wider AS. In the proposed structure, the feed probe is not directly connected to the antenna element but through a radial cavity so that the dominant radiator does not exist, which provides suitable condition for Rayleigh fading environment. And its possibility as spatial fading simulator was shown by authors[8][9]. However, the evaluation for Rayleigh fading and angular spread (AS) was not enough so that, in this paper, we show several computer simulation results on stochastic spatial characteristics of simulator.

In chapter 2, we introduce concept, methodology, design and characteristic of CECA. In chapter 3, we show the scenario of computer simulation and investigation on the terms to verify Rayleigh fading and AS. And next, computer simulation results will be shown on each terms and make a conclusion.

\section{CAVITY-Excited Circular ARRAY (CECA)}

\section{A. Concept and Specification}

Figure 1 shows fundamental notions of CECA. In this example, the array is composed of six inductance-loaded element arranged concentrically for structural symmetry, and one feed pin at the center of the radial cavity. In appearance, the inductance-loaded elements seem to be parasitic elements, but, in this structure, contrary to the ESPAR antenna, these elements are not the parasitic elements in the sense that they are fed via the radial cavity. The radial cavity may be regarded as a feed circuit and the elements above the radial cavity are fed through the radial cavity with pins connected to the reactance elements.

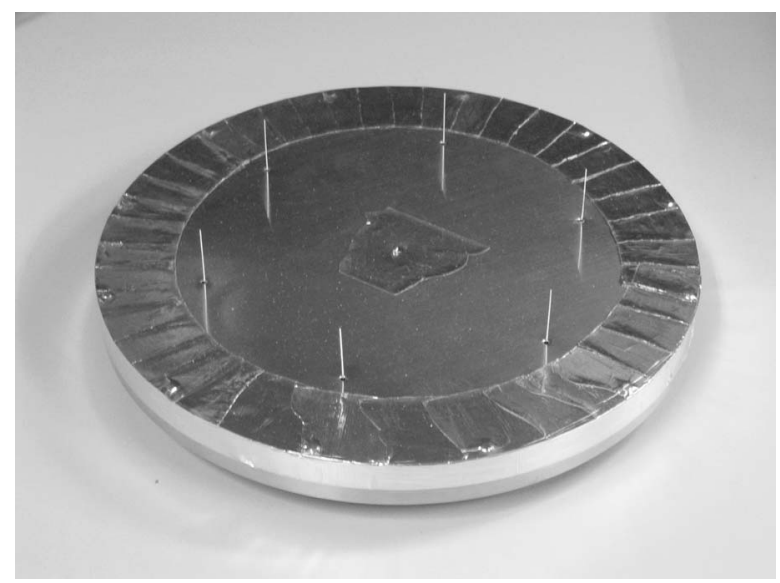

Fig. 3. Fabrication

Fading and AS that should be achieved Rayleigh fading channel can be realized as follows. In the case of fading, each of the fed elements has different phase due to the varied excitation coefficient by controlling the load impedance as well as the generation of higher order modes in the radial cavity. This quasi-random phase generates the Rayleigh fading. In other words, six array elements work as the local scatterer. Besides, ESPAR antenna produces Rayleigh fading only under specific condition. Contrary to this, CECA has a lot of flexibility because no direct wave exists. For AS, this structure is advantageous in the sense that the excitation strength is almost independent of the size of the radial cavity, since elements are fed by the radial cavity and not by the proximity coupling. Therefore, this structure has a wider AS than what an ESPAR antenna can achieve. Selecting the appropriate size of the radial cavity and array would easily control the AS. Figure 2 shows the structure and coordinate of CECA. Table I defines dimensions of CECA including distance of antenna element from wall and center $r s$, element length $l$, radial cavity height $h$. Figure 3 shows the fabricated CECA.

TABLE I

Structural Dimensions of CECA

\begin{tabular}{|c|c|c|c|c|}
\hline$r$ & $s$ & $l$ & $h$ & \\
\hline 0.675 & 0.297 & 0.197 & 0.096 & $\lambda(2.41 \mathrm{GHz})$ \\
\hline 8.4 & 3.7 & 2.45 & 1.2 & $\mathbf{c m}$ \\
\hline
\end{tabular}

\section{B. Parameters}

We have made use of the Method of Moment (MoM) simulator, FEKO[10] as numerical analysis method. The key parameters are the height of circular cavity, radius of CECA, position of array element. We first design and analyze CECA without varactor.

1) Hight of Circular Cavity: As we have mentioned in the previous section, radial cavity plays the roll as feed circuit. Therefore, the height of radial cavity is designed as low as possible so that the current distribution on array elements 
inside the cavity will almost be uniform on the z-axis unless the return loss becomes extremely worse , and it provides convenience to design CECA.

2) Radius of CECA: We design that the fabricated CECA works on the $T M_{0 n 0}$ The feed post of CECA connected to the upper wall of cavity at the center and low height of cavity make CECA work in the $T M_{0 n 0}$ mode. The fabrication works in $T M_{020}$ mode and its field satisfy follow equations. They are

$$
\begin{aligned}
E_{\rho} & =0 \\
E_{\phi} & =0 \\
E_{z} & =\sqrt{\frac{1}{\pi h}} \frac{J_{0}\left(\rho_{0 n} r\right)}{a J_{1}\left(\rho_{0 n}\right)} \\
H_{\rho} & =0 \\
H_{\phi} & =-\sqrt{\frac{1}{\pi h}} \frac{J^{\prime}\left(\rho_{0 n} r\right)}{J_{1}\left(\rho_{0 n}\right)} \\
H_{z} & =0
\end{aligned}
$$

, where $r, h$ and $\rho_{m n}$ represent distance of array element from center, height of CECA and the $n$th roots of $J_{m}=$ 0 respectively. We obtain a suitable radius of CECA with boundary condition,

$$
\begin{aligned}
E_{z} & =0 \\
r & =\frac{\rho_{0 n}}{\rho_{0 n^{\prime}}},\left(n, n^{\prime}=1,2,3, \ldots\right) \\
& =0.4357 \lambda, 1 \lambda, 1.5678 \lambda, \ldots(n=2)
\end{aligned}
$$

. We choose $1 \lambda$ due to proper calculation time. It has an error of $2.8 \%$ compared to simulation.

3) Position of Array Element: We locate array elements at the point of the strongest current so that energy is able to emit at its maximum. It shoud also be a null point of $H$ field. Namely,

$$
\begin{aligned}
H_{\phi} & =0 \\
r & =\frac{\rho_{1(n-1)}}{\rho_{0 n^{\prime}}},\left(n, n^{\prime}=1,2,3, \ldots\right) \\
& =0.694 \lambda, 1.271 \lambda, \ldots(n=2)
\end{aligned}
$$

We choose $0.694 \lambda$ due to proper calculation time. It has an error of $0.9 \%$ compared to simulation. This is very important parameter determining AS which will be mentioned next chapter.

4) Length of Array Element: As we have mentioned in the previous chapter, the circular cavity feed power to array elements. We manipulate array elements as monopole antenna, which inform that the length of array element in out of cavity may be $\lambda / 4$.

\section{Reactance Domain}

In this part, we show the characteristics of CECA on the reactance domain with simulation results[11]. It is necessary to verify the variation of phase on array elements in case of loading varactors at all array elements. We suppose that the capacitance of the varactor has been varied in the range of $0.5 p F-9 p F$, which correspond to $-132.63 \Omega--7.37 \Omega$.

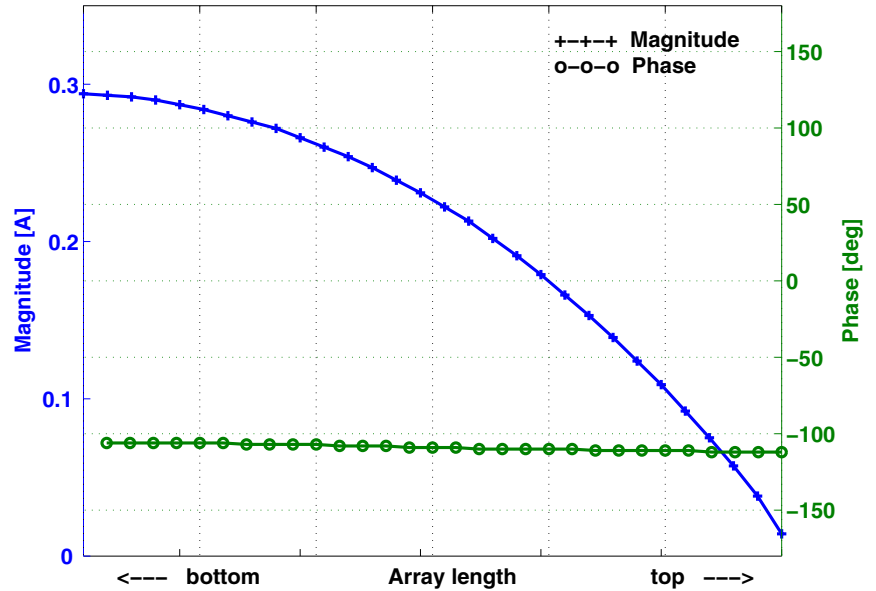

Fig. 4. Current distribution on array elements - when same varactors are loaded

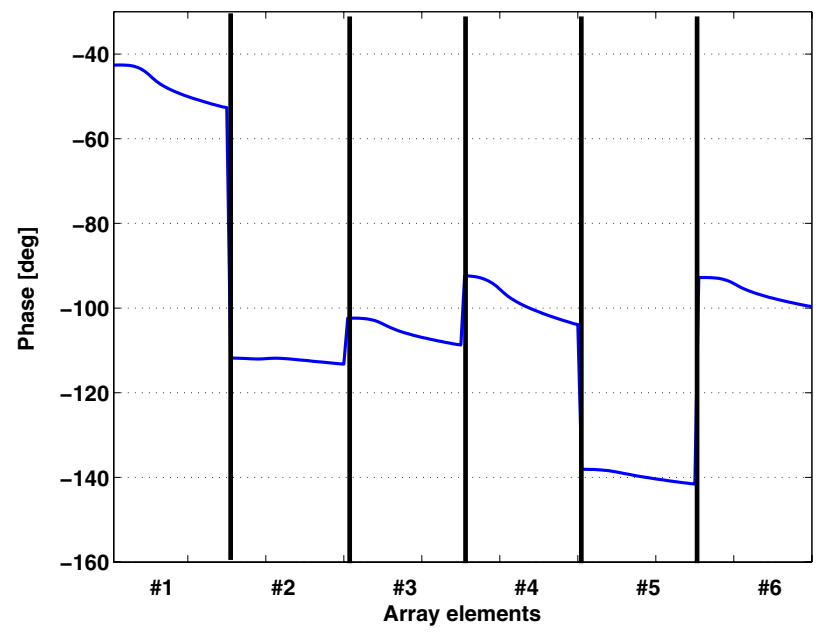

Fig. 5. Phase on each array elements - when random varactors are loaded

However, phase on array elements vary in more complicated manner than this value because of mutual coupling between array elements both inside and outside of the cavity. We compare two cases - fixed reactance and random reactance of varactor. Figure 4 shows current distribution on array elements when all array elements are connected to $4.2 \mathrm{pF}$ varactors. All the elements have the same current distribution because CECA has a symmetric structure. Next, we change the reactance of the varactor at random. Figure 5 shows an example of the current distribution of the cavity when $0.5 \mathrm{pF}, 9 \mathrm{pF}, 4.7 \mathrm{pF}, 1$ $\mathrm{pF}, 2.8 \mathrm{pF}$ and $7.3 \mathrm{pF}$ are respectively loaded and the phase values on the array elements varying. We will show later that this random phase variation shall cause the spatial Rayleigh fading.

\section{FADing Simulation}

We obtain impedance $\left[Z_{c}\right]$ by simulation when the varactors have not been loaded.

$$
\left[Z_{c}\right][I]=[V]
$$




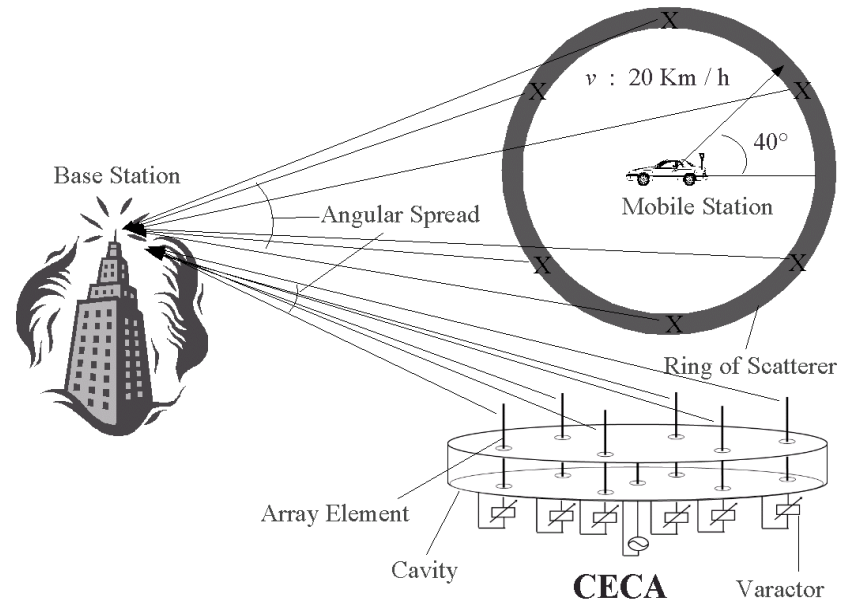

Fig. 6. Scenario

where $\left[Z_{c}\right],[I]$ and $[V]$ denote impedance matrix in the cavity, current vector defined at the elements inside the cavity and voltage vector between the elements and the lower plate of the cavity, respectively. When varactors have been loaded, the impedance $Z_{v a}$ is added to impedance $\left[Z_{c}\right]$ to obtain the current distribution. When the varactors are connected,

$$
\left[Z_{c}+Z_{v a}\right][I]=\left[V_{0}\right],
$$

where

$$
\left[Z_{v a}\right]_{n m}=-j \frac{1}{2 \pi f C_{m}},
$$

$V_{0}$ is the external applied voltage, while $f$ and $C_{m}$ denote the frequency and the capacitance of the $m$ th varactor respectively. In fact, we have to make use of the impedance of the upper part of the array element for simulating wave propagation. But since there is only a small difference in phase between the upper and lower parts of the array elements, we make use of the impedance of the lower part of array element instead of upper part's. In simulation, the Monte Carlo method is applied in order to make random local scatterers of array elements by random choice of the varactor reactance.

\section{A. Scenario}

In Lee's model, scatterers are evenly spaced on a circular ring about the mobile as shown in Fig.6[12]. In the same way, the array elements of CECA compose effective scatterers within the region which correspond to the upper area made by the position of the array element. The position of the array elements are determined by the cavity mode. Namely, the mode of the cavity decides the area of effective scatterers and its radius affects AS. While the mobile station is moving, the initial phase from scatterers can be simulated by controlling the variable inductor which is loaded to the array elements.

We assume that the mobile station moves with direction of $40^{\circ}$ and $20 \mathrm{~km} / \mathrm{h}$, and we simulate only 'short-term fading' under 'narrow-band assumption', which means that we do not consider time delay, which will be implemented in another work.

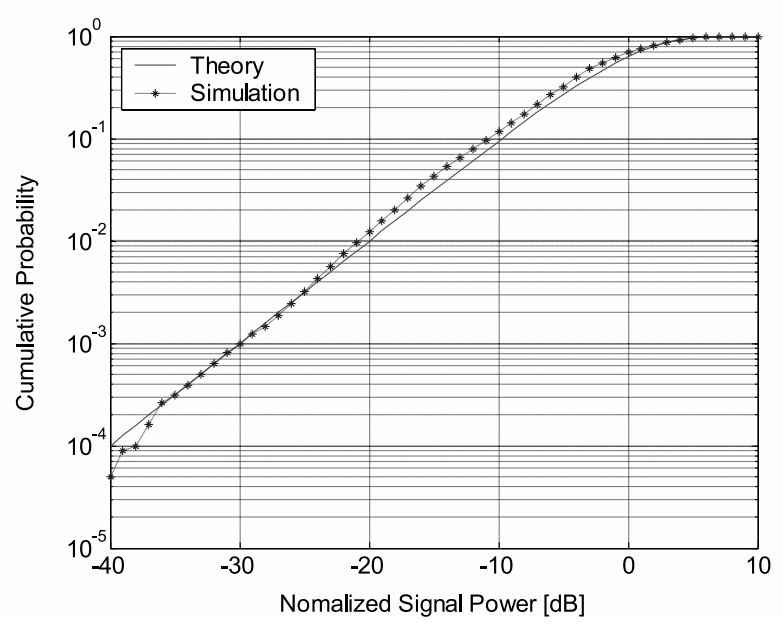

Fig. 7. Cumulative Probability of Fading Signal Power

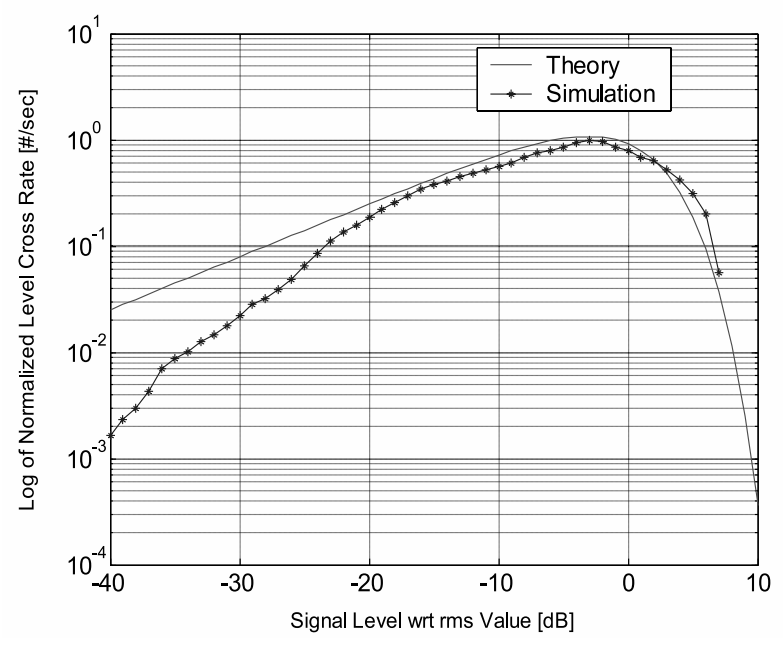

Fig. 8. Level Cross Ratio

\section{B. Spatial Stochastic Charateristics}

As mentioned above, to evalute antenna arrays at base station, we consider the fading phenomenon and angular spread. The power of the signal is Rayleigh distributed while AS depends on the height of the base station antenna[13].

1) Rayleigh Fading: As investigating the power cumulative density function (CDF), as shown in Fig. 7, we see that the power of received signal is close to a Rayleigh fading signal. Since we know that the received signal does not have a complex-Gaussian distribution, more investigation is necessary to confirm that this simulator can be useful for performance evaluation. Figures 8 and 9 show the received signal level crossing rate (LCR) and average fade duration (AFD). Figures show that though received signal is not a Rayleigh fading signal, it is so enough to evaluate performance of antenna array.

We have come to the conclusion with a well-founded conjecture as shown by the simulation results that this simulator genenerates the quasi-Rayleigh fading signal which is enough 


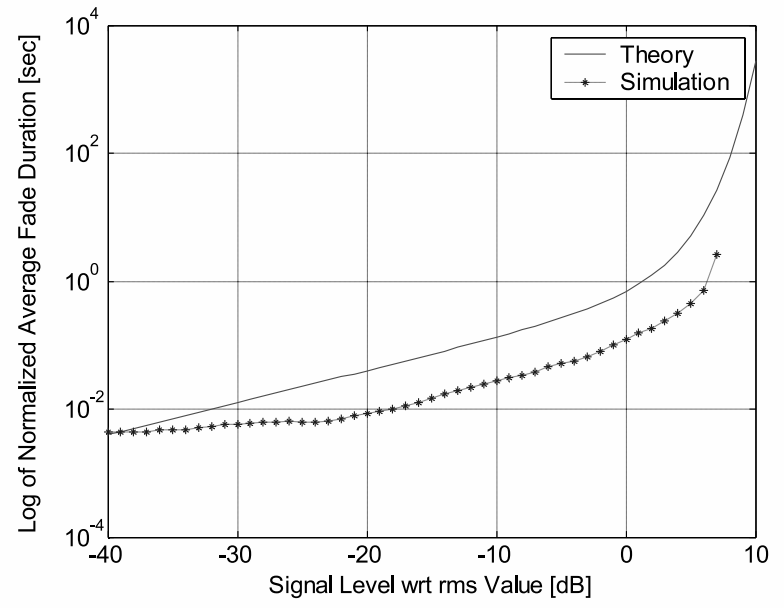

Fig. 9. Average Fade Duration

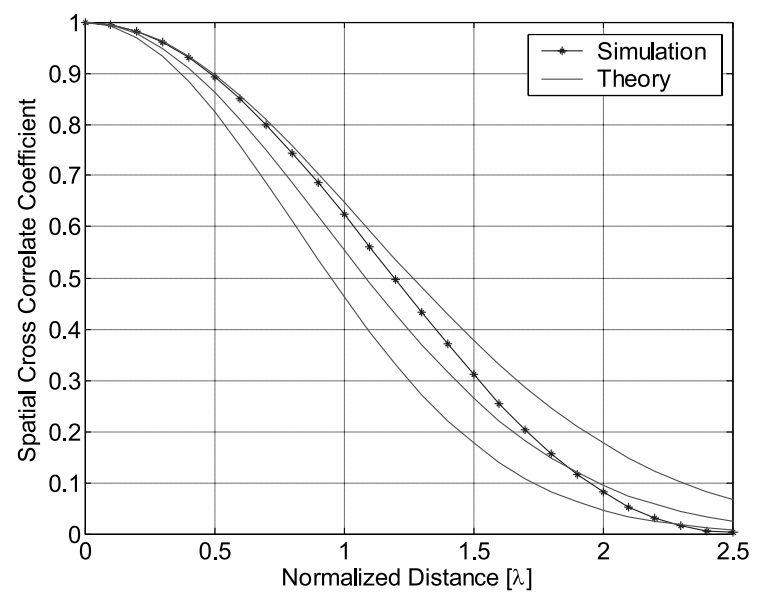

Fig. 10. Spatial Cross Correlation

to evaluate an antenna array.

2) Angular Spreading: The typical method to evaluate AS is to investigate on its spatial cross correalation. Figure 10 shows the simulated spatial cross correlation coefficient. Supposing that the power azimuth spectrum has a Gaussian distribution, AS ranges from $6^{\circ}$ to $8^{\circ}$ defined by its standard deviation. As mentioned above, AS is dependent on the height of the base station antenna and does not have a known fixed quantity. Controlling the radius of the cavity and distance between receiver and transmitter, we obtain several AS.

\section{Conclusion}

A cavity-excited circular array based on ESPAR antenna used as a spatial fading emulator has been proposed and its features and design methodology and measurements on the fabricated CECA are presented. We showed the computer simulation results to evaluate CECA as a spatial fading simulator for a base station antenna array. Though the generated signal is not Rayleigh faded, we found that it is still useful to evalute antenna arrays through several simulations. AS can be controlled by the mode of CECA or the distance between receiver and transmitter so that we can obtain several AS varying with the height of the base station antenna.

\section{ACKNOWLEDGMENT}

The author would like to thank Dr. I. Ida for his useful comments and suggestions.

\section{REFERENCES}

[1] W.C. Jakes, "Microwave Mobile Communications," John wiley Sons Inc., NewYork, 1974.

[2] U. Martin, "Modeling the Mobile Radio Channel by Echo Estimation," Frequenz, No.48, vol.9-10, pp. 198-212, 1994.

[3] T.S. Rappaport, "Wireless Communications," Prentice Hall, New Jersey, 1996.

[4] H. Arai, "Field Simulator for Rayleigh/Rician Fading Reproduction," 2000 IEEE AP-S International Symposium, pp. 1552-1555, Jul. 1996.

[5] R. Yamaguchi, "Fading generator using moving scatters," Proc. IEICE Gen. Conf.98', B-1-23, 1998.

[6] T. Ohira, "Electronically steerable passive array radiator(ESPAR) antennas," 2000 IEEE AP-S International Symposium, pp.922-925, Jul. 2000.

[7] K. Sakaguchi, A.L.S. Neves, J. Takada, K. Araki, and T. Ohira, "Spatial Fading Emulator Using an ESPAR antenna Structure," Proc. of International Symposium on Wireless Personal Multimedia Communications(WPMC'01), vol.2, pp.489-492, Sep. 2001

[8] C. Park, J. Takada, K. Sakaguchi, and T. Ohira, "Analysis of a RadialCavity-Excited ESPAR Antenna,” IEICE Tech. Rep., MW2003-122-150, pp.17-20, Sep. 2003.

[9] C. Park, J. Takada, K. Sakaguchi, and T. Ohira, "Spatial Fading Emulator using Cavity-Excited Circular Array based on ESPAR Antenna," accepted for International Symposium on Antenna and Propagation(ISAP'04), Aug. 2004.

[10] EM Software \& System, "FEKO," http://www.feko.co.za, Stelilenbosch, South Africa, 2001.

[11] T. Ohira, "Reactance Domain Signal Processing in Parasitic-Array Antennas (invited)", Asia-Pasific Microwave Conf., APMC2003, WD61, 1, pp290-293, Seoul, Korea, Nov. 2003.

[12] R. B. Ertel, P. Cardieri, K. W. Sowerby, T. S. Rappaport, and J. H. Reed, "Overview of spatial channel models for antenna array communication systems," IEEE Personal Commun., vol.5, pp.10-21, Feb. 1998.

[13] Klraus I. Pedersen, Preben E. Mogensen, Bernard H. Fleury, "Spatial Channel Characteristics in Outdoor Environments and their Impact on BS Antenna System Performance," IEEE Proc. Vehicular Technology Conference(VTC‘98), vol.2, pp.719-723, May. 1998. 